\title{
La biblioteca híbrida: ¿un paso hacia el acceso universal a la información?
}

\author{
Georgina Araceli Torres Vargas \\ Centro Universitario de Investigaciones Bibliotecológicas \\ Universidad Nacional Autónoma de México, 04510 \\ México D .F., Tel: (525)623-03-61 \\ E-mail: arageo@yahoo.com
}

\begin{abstract}
RESUMEN
Se aborda la idea de biblicteca híbrida, término que comienza a mencionarse con frecuencia en la literatura especializada para referirse a la biblioteca en la cual podría permitirse un acceso universal a la información. Al igual que la biblioteca digital o la virtual, este concepto se refiere a una alternativa para alcanzar la universalidad en el contexto tecnológico actual, pero parece prometer mayores alcances al tomar en cuenta soportes de información tradicionales. Por tal razón se analiza en qué consistiría la biblioteca híbrida y de qué manera puede favorecer la construcción de una biblioteca total.

Palabras clave: Biblioteca híbrida, Acceso universal a la información, Bibliotecas del futuro

THE HYBRID LIBRARY: A STEP CLOSER TO UNIVERSAL ACCESS TO INFORMATION? Georgina ARACELI TORREs-VARGAS

\section{ABST RACT}

This article offers an approach to the idea of the hybrid library, a term which has emerged recently and is now used frequently in the literature to describe a library that would allow universal information access. Not unlike the virtual or digital library, this concept emerges as an altemative route to achieving universal information access, yet it appears to promise a more comprehensive scope. With this in mind, the components of a hybrid library are analyzed as well as the conditions which would favor the establishment of a "total library."

Key Words: Hybrid Library, Universal Information Access, Library of the Future
\end{abstract}

Trabajo recibido el 2 de diciembre de 1999

*

Trabajo aceptado el 25 de enero de 2000

\section{INTRODUCCIÓN}

T a creación de una biblioteca en donde esté contenido todo el conocimiento re$\_$gistrado es una quimera que por siglos ha tenido el hombre. Este sueño es evidente en los restos de la biblioteca de Ebla, la casa de la vida egipcia, las bibliotecas monacales e incluso en las bibliotecas nacionales. 
En una primera etapa se pretendió que la biblioteca fuera el sitio en donde se preservara la memoria de la humanidad. El modelo más representativo dentro de esta concepción fue la biblioteca de Alejandría.

También en la obra de importantes escritores pueden encontrarse diferentes alusiones a esa biblioteca universal. En este caso una de las obras más citadas es La biblioteca de Babel, de Borges. ${ }^{1}$

Y también Jünger en su obra Heiópdis se refiere a estaidea de totalidad en el acceso a la información, cuando al describir un artefacto usado por uno de los protagonistas de su novela, habla en el sentido de que el orden enciclopédico abarcabainmensos dominios, con sutilesy perfectas subdivisionesen donde además se añadíala documentación escrita procedente de los catálogos de museos y de la bibliografía. ${ }^{2}$

Jünger hace alusión a la idea de universalidad que se mantenía frente a los documentos impresos: la creación de bibliografías exhaustivas, universales. Idea que durante mucho tiempo recibió el apoyo de diferentes autores que encaminaron gran parte de sus esfuerzos a la formación de catálogos o repertorios en los que se registrara la información generada en el mundo. Vista así, la universalidad se centraba en el registro de los documentos, a través de grandes catálogos.

Con la aparición del concepto de biblioteca nacional se persiguió lo mismo que en el modelo planteado en la biblioteca de Alejandría, con la diferencia de que cada una de estas bibliotecas tendría además que compartir o difundir su acervo a través de una bibliografía nacional elaborada por la misma institución. En esta visión el fin de la formación de colecciones nacionales sería la constitución de un control bibliográfico universal.

Bajo esta idea de universalidad, diferentes organismos internacionales como IFLA, FID y UNESCO, han concebido programas encaminados hacia la búsqueda de mecanismos que permitan la cooperación internacional. Todos ellos ponen énfasis en la conservación del patrimonio documental, en la normalización y en el uso de recursos tecnológicos para intercambiar registros bibliográficos.

En la actualidad se piensa que mediante la creación de documentos electrónicosy su disposición en redes de telecomunicación se tendrá acceso universal a los registrosy a los textos. Seasevera que la tecnología delainformación será una herramienta útil para acceder a la información, y que terminará con las barreras temporales y espaciales, pues podrá hacerse uso de los documentos digitales a cualquier hora del día y desde cualquier sitio. ${ }^{3}$

Se piensa que a través de una biblioteca virtual, digital, o electrónica, el acceso universal tendrá nuevas características y que serán diferentes a las que se observan en el medio impreso.

1 Jorge Luis Borges. "La biblioteca de Babel". En: Ficiones/ Jorge Luis Borges. - Madrid, España: Alianza, 1998.

2 Cfr. Ernst Jünger. Heićpolis visón retrospetiva deuna audad- Barcelona : Seix Barral, 1998.

3 Ellen W. McD onell. "Knowledge transfer and the virtual library" En: FID NensBulleinv. 44 (jul-ag 1994) n. 7-8. p. 139-141. - p. 140. 
Sin embargo, en opinión de algunos autores, la biblioteca digital se basa en el uso de documentos digitales y excluye a los impresos, aspecto que seguiría limitando el acceso a la información. Frente a estas consideraciones ha surgido lugar a un nuevo término que alude a una nueva alternativa de biblioteca: la biblicteca híbrida.

\section{EL ACCESO A LA INFORMACIÓN}

A pesar de que en los artículos especializados se utiliza con frecuencia la palabra acceso los autores que la usan no suelen definirla. Sin embargo con base en el uso que se le da en los diferentes contextos es posible desprender dos acepciones:

\section{Se puede entender como el proceso de "aproximación" a la información} documental. 4

Visto así, se refiere a la identificación de los documentos, sea a través de catálogos, repertorios bibliográficos o bases de datos. Representa un acercamiento a la información en el sentido de que es la instancia que permite descubrir aquellos materiales que probablemente contienen la información requerida y, en algunos casos (por ejemplo, en el catálogo de una biblioteca), nos indica dónde se localiza el documento.

A través de los registros que conforman un catálogo, el usuario puede encontrar un libro específico empleando diferentes puntos de acceso. El catálogo de tarjetas es una gran aportación parala organización de información, al permitir describir un solo libro bajo diferentes aspectos.

D esde su inicio el catálogo de tarjetas ofreció múltiples puntos de entrada, pero la creciente generación de documentos obligó a desarrollar esquemas clasificatorios para, en principio, ubicar físicamente al libro. Posteriormente estos sistemas ofrecerían la ventaja de poder agrupar y relacionar las obras.

Como las colecciones de las bibliotecas eran pequeñas se desarrollaron sistemas de clasificación propios; no obstante el crecimiento de las colecciones pronto hizo necesaria la construcción de sistemas de carácter universal. Estos sistemas guardaron una estrecha correspondencia con la idea de acceso que prevalecía en el medio de los impresos, es decir, con la tendencia a la construcción de grandes catálogos que registraran toda la información generada en el mundo.

En los sistemas clasificatorios universales subyacían las bases del enciclopedismo, según las cuales los esquemas podían reflejar los conocimientos distribuidos sobre la faz de la tierra y exponer un sistema general, que los hombres podrían ir cubriendo con los saberes desarrollados en siglos posteriores. El proyecto del

4 En este caso se considera al documento como fuente de información, que de acuerdo con López Y epes, es un soporte que contiene una información potencialmente transmisible en el espacio y en el tiempo, actualizable y que permite alcanzar un nuevo conocimiento o tomar una decisión acertada (Cfr. José López Y epes. Loscaminos delainfomadón: cómbluscar, sdeccionaryarganizarlasfuentesde nuestra doumentacón personal. - Madrid : Fragua, 1997. - p. 44). 
enciclopedismo pretendía, entre otras cosas, la acumulación de saberes a través de una sola obra; la enciclopedia sería el libro de los libros, el registro de la totalidad de saberes y la reconstrucción de la memoria y el orden del mundo. ${ }^{5}$

En el contexto de los documentos impresos, tanto los sistemas de clasificación como la idea de biblioteca mantenían semejanzas con esta idea, pues se consideraba que la biblioteca ideal sería aquella en la que se cucumulara toda la memoria del mundo.

En los Estados Unidos, la Biblioteca del Congreso emprendió el desarrollo de un sistema que fuera lo suficientemente preciso como para permitir la clasificación deuna colección numerosa. La clasificación de la Biblioteca del Congreso deWashington es un claro intento de exhaustividad. Pero debido a la especialización de los saberes, actualmente esta biblioteca construye otro sistema para ordenar los libros de manera más efectiva, bajo la idea de universalidad.

\section{2. "Entrar" a la información documental.}

La segunda acepción de accesoimplica tanto la disponibilidadcomo el usodelainformación. Existe disponibilidad cuando:

* Se tiene el documento físicamente al alcance. Por ejemplo, existe disponibilidad cuando el libro previamente identificado se encuentra en el estante, listo para ser usado.

* Se puede obtener el documento mediante los servicios de información. El desarrollo histórico de las bibliotecas nos muestra cómo en un principio se daba la práctica del acceso restringido, que limitaba la consecusión de los documentos a sólo ciertas clases sociales. En relación con esta situación, la idea de biblioteca pública significó una apertura, pues pretendía que toda persona tuviera el derecho de acceder a los servicios de información. Este aspecto de la disponibilidad es el que se relaciona con los servicios de información y más directamente con el concepto de préstamo. Algunos autores sostienen que históricamente el préstamo interbibliotecario ha sido considerado como obligatorio por las bibliotecas, y que al permitir el acceso a los materiales de otras colecciones bibliotecarias se convirtió en uno de los servicios más básicos que proveen las bibliotecas.

Por lo que respecta al uso:

* Laprimera condicionante para que el sujeto puedausar lainformación es saber leer: poder descifrar los signos que están inscritos en el documento. Por esa razón una de las principales barreras para acceder a la información es el analfabetismo. O tra barrera es la idiomática.

* Junto con la lectura, se requiere poder interpretar el texto. D e acuerdo con lo que señala Miguel Angel Rendón:

5 Jean-Marie Goulemot. "Bibliothéques, encyclopédisme et angoisses de la perte : l' exhaustivité ambigüe des Lumiéres”. En: Lepancirdesbiblidhéques: lamémiredeslivesenOcident/ sausladiretion deMarcBaratin \& distianJacdb - París, Francia : Albin Michel, 1996. - p.290-291. 
[...]el paso del símbolo a la información es unaactividad que se realizay se repite cada vez que se lleva a cabo la estructuración de datos por diferentes sujetos o por el mismo sujeto. Si no se conocen las reglas de estructuración, el documento puede estar ahí enfrente de nosotros y no decir nada. 6

El individuo interpreta a partir de su contexto y lo que condiciona su acceso o no a cierta información es su estructura de pensamiento y el entorno en el que él se desarrolla. Buckland menciona que además de la identificación y la disponibilidad, también el aspecto cognitivo es una de las barreras que dificultan el acceso a la información. ${ }^{7}$

Sin embargo la condición de uso implica factores externos al control de los profesionales de la información. Por tal razón, puede aseverarse que la aproximación y la disponibilidad son los medios a través de los cuales la documentación puede permitir el acceso a la información.

Las formas de acceso hasta aquí mencionadas, se manifiestan de manera particular en cada tipo de biblioteca. Por ejemplo en el contexto de la biblioteca del futuro ${ }^{8}$ se espera tener un acceso que corresponda con las condiciones que se den en la sociedad de la información.

\section{EL ACCESO A LA INFORMACIÓN EN LA BIBLIOTECA DEL FUTURO}

El sueño de lograr un sistema de organización y almacenamiento del conocimiento humano universal - que se concentre en un compendio como la Enciclopedia o en una Biblioteca Universal- se agudizó con el rápido desarrollo del saber científico que acompañó al esfuerzo militar de las dos guerras mundiales9.

En TheGutenbagGalaxy, Marshall McLuhan presagió que las formas de conocer implantadas con la invención de la imprenta, se hallaban a punto de ser sustituidas por una forma más global de percibir y comprender a través de imágenes televisivas u otros dispositivos electrónicos ${ }^{10}$. Tal opinión fue retomada por diferentes autores, defensores de una apocalíptica de la biblioteca, quienes se apresuraron a aseverar su pronta desaparición dado que su forma estática no le permitiría amoldarse a los desafíos planteados por los medios electrónicos.

6 Miguel Angel Rendón Rojas. Bases teéicas yfilosóficas dela biblidtedogóa - México : UNAM, CUIB, 1998. - p. 112.

7 Michael Buckland. Libraryservicesin theeryand context. D isponible en: http:/ / sunsite.berkeley.edu/ literature/ library/ services/

8 A veces denominada como biblioteca digital, biblioteca electrónica, biblioteca virtual, etcétera.

9 Francisco Javier G arcía Marco. "Vannevar Bush, el hipertexto y el futuro del documento". - En Tendanias deinvesticaacón en documentación: adas dd seminario/ Jesús Tramullas, ed. - Zaragoza : Universidad de Zaragoza, 1996. - p. 194.

10 Umberto Eco. "El porvenir de los libros". - En 25CongresodelaUniónIntemacianal deEditares - Barcelona : ¿? 1996. - p. 4. 
Con base en la idea de la sociedad de la información, esta concepción tomó un leve giro: la biblioteca no desaparecería, pero sí cambiaría su naturaleza11. En todo caso, lo que estaba en vías de extinción eran los medios impresos.

Es así como Lancaster, junto con otros, reiteraron en sus escritos que los adelantos en computación y en tecnología de la comunicación, harían que la impresión en papel cediera totalmente el paso a la electrónicay a que se produjeran solamente publicaciones legibles a máquina. ${ }^{12}$ Con esta teoría denominada sociedadsin papd se argumentaba que la naturaleza de la biblio teca cambiaría a causa de las nuevas formas de publicación de las obras, puesto queno se contaríaya con colecciones impresas.

Con estas expectativas sobre la publicación electrónica y el uso de las futuras redes de comunicación como medio para su diseminación, surgieron nuevas concepciones en torno ala biblioteca. Labiblioteca del futuro es, a partir de entonces, un tema de estudio que se enfoca desde diferentes aristas: el papel del profesional delainformación; las nuevas formas en la organización y el manejo delainformación, son sólo algunas.

Esta biblioteca se ve como una biblioteca sin paredes, donde todos los textos pueden ser ubicados, reunidos y leídos. En el mundo de la recuperación electrónica, hecha posible por las comunicaciones digitales y electrónicas, los textos no son prisioneros de su origen físico o su característica material. Separados de los objetos, los textos pueden ser transmitidos. Ahora el catálogo de todos los catálogos, que contenga idealmente toda la producción escrita, se corresponde con el acceso electrónico a todos los textos disponibles universalmente para su consulta. ${ }^{13}$

Según Roger Chartier, suponiendo que todos los textos existentes hayan sido convertidos en textos electrónicos, la disponibilidad universal del patrimonio escrito sería posible. Todo lector, con la condición de que esté conectado frente a un puesto de lectura con la red informática que asegure la distribución de los documentos, podrá consultar, leer o estudiar cualquier texto, cualesquiera que sea su forma y su localización originales. ${ }^{14}$ El desarrollo de nuevas tecnologías, sostiene este autor, permitirá que en el futuro los textos existan de manera electrónica, y sean compuestos en la computadora y estructurados mediante procedimientos telemáticos antes de llegar a un lector, que los recibirá en una pantalla. D e esta forma la biblioteca del futuro se convierte en una opción ideal, a la vista de algunos autores, porque en comparación con la biblioteca física basada en impresos, provee información de manera más rápida y eficiente. ${ }^{15}$

11 Aunque hay que señalar que aún hay autores que apuestan por su desaparición, sobre todo basándose en la premisa de que una biblioteca virtual podría permitir que el usuario dejara de asistir a un sitio concreto y de solicitar ayuda a un bibliotecario para satisfacer sus necesidades de información.

12 W. Lancaster,. Tonard paperessinfomation system - Nueva Y ork : Academic Press, 1978.

13 Roger Chartier. "Libraries Without walls". En Futurelibranies/ Howard Bloch and Carla Hesse, eds. - Berkeley : University of California Press, 1995. - p. 48.

14 Ibid. - p. 260.

15 Walt Crawford. Futurelibranies: dreams, madness\& reality. - Chicago : American Library Association., 1995. - p. 133. 


\section{El acceso en el CONTEXTO ACtual}

Basándose en ideas como las anteriormente señaladas, muchas bibliotecas y programas han efectuado ciertos cambios para lograr un acceso universal a la información. Una de las primeras acciones fue la iniciativa para la creación de una Infraestructura Nacional de Información (NII, por sus siglas en inglés), en los Estados Unidos, a través de la cual se propuso y discutió la pertinencia de:

* O frecer un acceso universal que promoviera un nivel democrático de participación civil.

* Dar libertad para comunicar una amplia y diversa información.

* Utilizar tecnologías que hicieran un trabajo altamente equitativo y de calidad.

Con la creación de una NII, se pretende que los servicios de información multimedia ${ }^{16}$ estén al alcance de cualquier persona y apoyen la salud, la educación, la recreación y la difusión de los servicios de información gubernamentales. Con la idea de crear una Infraestructura Nacional de Información, se plantea también el concepto del servicio universal, el cual se detalla en el acta de telecomunicaciones de 1996 de los Estados Unidos. ${ }^{17}$

Este servicio contempla a la libertad como principio básico; de hecho es ésta la idea principal que se tiene sobre el acceso en la llamada era de información. Se cree que gracias a las autopistas de la información todos los humanos tendremos un acceso igualitario a este recurso. Tal es la nueva ideología imperante y que difunden los defensores del mercado global y los partidarios del libre flujo de datos.

El pensamiento de universalidad en el medio digital se puede bosquejar de la siguiente manera:

* Todos los datos, información y conocimiento registrado sobre todos los temas deben estar disponibles en todo momento y desde cualquier lugar.

* Cualquiera debe poder hacer uso efectivo de todos los datos e información.

* Las bibliotecas deben dejar de existir como entidades físicas y los impresos como medio de publicación y distribución. ${ }^{18}$

Sin embargo aunque existen estos buenos deseos, el acceso universal que se persigue es todavía difícil de realizar debido a limitantes de diversa índole que merecen ser analizadas aparte.

Pese a ello en el contexto actual se continúa trabajando a favor de la construcción de bibliotecas que ofrezcan documentos a través de medios tecnológicos. D e esta forma se dedican grandes esfuerzos y recursos a la digitalización de los documentos impresos, pues uno de los objetivos más perseguidos es poder contar a futuro con

16 Se refiere sobre todo al acceso a la información dispuesta en forma de video, audio y datos que se encuentran en las redes de telecomunicación.

17 Cfr. Patricia Aufderheide. Communications pdigy and the public interest : the tdecommmications Ad of 19996. - New York : Guilford Press, 1999. - p. 46.

18 Ibid, p. 87. 
colecciones completamente digitalizadas que se pongan a disposición de los usuarios por medio de las redes electrónicas.

Por lo que toca a la digitalización de textos impresos, los vacíos jurídicos sobre derechos de autor y sobre la publicación de las obras en formato digital plantean todavía problemas espinosos. D ebido a ello, además de otros factores, casi todas las bibliotecas se están limitando a convertir a este formato los materiales publicados antes de 1920. La Biblioteca del Congreso por ejemplo, ha digitalizado millares de fotografías de la Guerra de Secesión, documentos del Congreso Continental y discursos de la Primera Guerra Mundial, pero no puede digitalizar Loqued vientosellevó por eso muchas de las obras que en origen se publicaron en impreso, no pueden estar en línea. 19

También el aspecto monetario impide que se digitalicen todos los registros universales. Son muchos los documentos que no están en forma electrónica, en realidad menos del cinco por ciento de la información y el conocimiento mundial registrado están digitalizados. ${ }^{20}$ Como sólo es posible digitalizar algunos de estos documentos, surge otra preocupación: ¿ A cargo de quiénes correrá la decisión de cuáles registros deben ser digitalizados, del gobierno o de las grandes corporaciones? A demás la conversión selectiva vendrá acompañada de la imposibilidad de acceder al material no convertido, por lo que ya muchos comienzan a considerar lanecesidad de preservar el material impreso valioso. De una situación similar es de donde se desprende la noción de biblioteca híbrida.

\section{BIBLIOTECA HÍBRIDA Y ACCESO}

Tochtermann, investigador del Centro de Estudios de Bibliotecas D igitales de la Texas A\&M University, señala que la colección de una biblioteca virtual consta de documentos digitales y recursos de Internet, pero debe complementar sus servicios con los de la biblioteca tradicional y explotar las ventajas de la tecnología. ${ }^{21} \mathrm{O}$ tros autores opinan lo mismo que Tochtermann y no consideran a los documentos digitales como los únicos materiales que deban constituir la biblioteca del futuro.

Richard D e G ennaro explica que la idea de biblioteca abierta no implica reemplazar la colección de libros con tecnología, sino que considera a las fuentes documentales como algo que puede incrementar su capacidad de disponibilidad en el futuro. Él describe una situación en la que las bibliotecas deben proveer acceso a un rango de diferentes medios, pero también expresa el ideal de una gran integración.22

19 Michael Lesk. "Bibliotecas digitales". - En Investigaciónycienaia - No. 28 (mayo 1997). - p. 52.

20 Walt Crawford. Op Cit, p. 90.

21 Klaus Tochtermann. A first steptonard commmicationin thevirtual libraies Disponible en: http:/ / www.cdsl. tamu.edu/ csdl/ publs/ klaus/ TecRepKlaus.html

22 Stephen Pinfield. Realizingthehibridlibrary. Disponible en: http:/ / mirrored.ukoln.ac.uk/ lis-lournals/ dlib/ dlib/ dlib/ octobre98/ 10pinfield.html 
Por su parte Chartier señala que la biblioteca del futuro debe ser el lugar en donde se pueda mantener el conocimiento y la comprensión de la cultura escrita en su forma original. Afirma que la representación electrónica de todos los textos no debe significar de ninguna manera la relegación, el olvido, o la destrucción de los objetos que los han producido. Más que nunca, quizá, una de las tareas esenciales de las grandes bibliotecas es recolectar, proteger y censar los objetos escritos del pasado. ${ }^{23}$

Algunos líderes bibliotecarios esperan que la biblioteca del futuro sea un híbrido donde se reúnan colecciones de libros y colecciones digitales. Para muchos ésta puede parecer una situación común en la actualidad, ${ }^{24}$ puesto que existen numerosas bibliotecas que contienen materiales de toda índole. Sin embargo, debe buscarse una alternativa para hacer que la coexistencia de impresos y documentos digitales se dé bajo el reconocimiento de las diferencias que cada entorno presenta. La labor será lograr una simbiosis entre lo digital y lo impreso, y esto llevará a enfrentar diversos retos, como la necesidad de implementar diferentes formas de organización.

Muchas de las recientes visiones sobre la biblioteca del futuro especulan con la desaparición del libro, pero la discusión no debe centrarse en ese aspecto. La información digital y la impresa representan dos ámbitos totalmente diferentes. Ninguna suple a la otra. La biblioteca híbrida no debe ser considerada como una etapa de transición sino de armonización entre estos dos medios.

Labiblioteca híbrida debe diseñarse para encauzar las tecnologías dentro del contexto de la labor bibliotecaria y explorar sistemas y servicios tanto en el ambiente impreso como en el electrónico. Esta biblioteca es muchas veces considerada como una fase entre la biblioteca convencional y la digital, pero por encima de eso, es, sobre todo, un modelo.

Labiblioteca híbrida explora eintegra sistemas y servicios en medios electrónicos e impresos, se propone darle acceso a los diferentes soportes de información, usando las tecnologías del mundo digital y los diversos medios de comunicación.

Esta imagen de biblioteca se acerca más a una biblioteca abierta que a una virtual o digital. Su relación con el acceso universal a los documentos se antoja más viable, en tanto que atendería realmente el universo de documentos.

La biblioteca híbrida debe implementar nuevos servicios, pero trabajar en el contexto de un sitio concreto. ${ }^{25}$ Claro está, sin perder de vista la relación mediante redes y el intercambio y uso de la información por medio de éstas.

En realidad el objetivo es desarrollar sistemas de información que permitan el acceso a esta colección compuesta por documentos impresos y digitales, para que todos los materiales puedan ser explotados. El valor de la biblioteca del futuro se verá

23 Chartier, Roger. "D el códicea la pantalla : las trayectorias de lo escrito". - En Sociedadyøarturaenla edadmodama: la altura combapropiación - México : Instituto Mora, 1995. - p. 263.

24 Andrew 0 dlyzko. "Silicon dreams and silicon bricks : the continuing evolution of libraries". En: LibraryTrends - Vol. 46, no. 1. - (summer, 1997). - p. 162.

25 Chris Rusbridge. Towards thehybridlibrary. Disponible en: http:/ / www.dlib.org/ dlib/ july98/ rusbridge/ o7rusbridge.html 
acrecentado en la medida que la colección de la biblioteca tradicional sea accesiblea través de laintegración de la representación de materiales digitales como la multimedia, datos geoespaciales, etcétera.

En este terreno ya se observan algunos intentos. Los cinco proyectos de e-Lib (instituciones y organizaciones del Reino Unido, dedicados al área de educación superior), ${ }^{26}$ comenzaron en enero de 1998, y aprovechan el desarrollo de la biblioteca híbrida en diferentes sentidos: desarrollando bases de datos, interconectividad, digitalización, interfaces de usuarios.

Uno de los elementos claves del cambio es la diversidad de bibliotecas. El reto es lograr una interoperabilidad entre ellas, esta interoperabilidad en las bibliotecas se puede considerar desde dos puntos de vista:

1. El uso común de herramientas e interfaces que permitan una uniformidad superficial en la navegación y el acceso.

2. La interoperabilidad semántica, que para poder ser intercambiada dependería de la similitud y consistencia del tipo de información (textual, audio, numérico, multimedia). Ello implicaría a los protocolos (tal como el HTML en Internet), los metadatos y el tipo de clasificación, lo que daría como resultado una única referencia global, que pudiera presentarse a través de los enlaces por red.

Lo ideal sería poder disponer de una infraestructura única que admitiera todo tipo de servicios y aplicaciones, pero habría que construirlay para ello sería necesaria la colaboración, regulación, estímulo y ayuda, de los países involucrados. También sería necesaria una estandarización de interfaces que permitiera la circulación de la información multimedia entre todos los actores. Junto con ello, deberáhaber diversos proveedores de:

$$
\begin{aligned}
& \text { * red } \\
& \text { - servicios } \\
& \text { - y contenidos }
\end{aligned}
$$

En este contexto la biblioteca misma podríajugar un papel importante. Una multitud de nuevos medios y el acceso rápido a las redes de cómputo revolucionaría nuestros conceptos sobre el libro, las bibliotecas, la investigación científica, la enseñanza-aprendizaje y el comercio, entre otros aspectos. La conjunción de libros y otros recursos en las bibliotecas debe ser una constante que no debe ser abandonada. La biblioteca tiene que continuar apoyando la diversidad. ${ }^{27}$

Aunque es cierto que la biblioteca no es capaz de cubrir por sí misma todas las necesidades de información ni puede preservar toda la cultura impresa, si se cooperara con ella podría construirse un mejor ambiente. Esta colaboración podría darse tanto a través de las redes como de los canales que se relacionan con el mundo de los impresos.

26 Para más detalle sobre estos proyectos, consultar en: http:/ / www.ukoln.ac.uk/ services/ elib 27 Walt Crawford. Op Cit, p. 178. 


\section{CONCLUSIONES}

Es claramente notorio que la biblioteca del futuro implica una serie de promesas sobre el acceso universal a los documentos, pero también es cierto que existen algunos obstáculos que podrían impedir que este modelo se concretara. Entre otras cosas, se nota ya una gran dificultad para digitalizar las obras que existen en forma impresa, puesto que esa labor de digitalización implica una labor titánica difícil de alcanzar con prontitud. A esto hay que añadir las limitantes de tipo jurídico que impiden digitalizar lo más reciente y el costo que implica esta labor. O tro aspecto importante que se debe considerar, es que los impresos no dejarán de existir a causa de la aparición de publicaciones electrónicas; necesariamente coexistirán el llamado mundo impreso con el mundo digital.

Situaciones como éstas hacen cada vez másclara la certeza de que muchos de los materiales impresos serán excluidos de la colección de la biblioteca del futuro, cuya visión se idealiza. Todo esto estaría reflejando una falsa universalidad en el acceso a la información.

Para decir que se cuenta con tal acceso, la biblioteca del futuro deberá contener tanto materiales digitales como impresos, pues la búsqueda de una universalidad se vería frustrada si se piensa que el acceso universal sólo puede darse a través del entorno electrónico.

En definitiva, hay todavía que resolver las diferentes barreras que se oponen a la construcción de una biblioteca mundial diseminada y uniforme que posea la totalidad de documentos de nuestra herencia humana. Una alternativa que se observa prometedora es la creación de entornos híbridos, por eso lo importante ahora es analizar cómo se puede hacer realidad esta idea de biblioteca que promete una total disponibilidad de la información, y cómo podemos aproximarnos a ella.
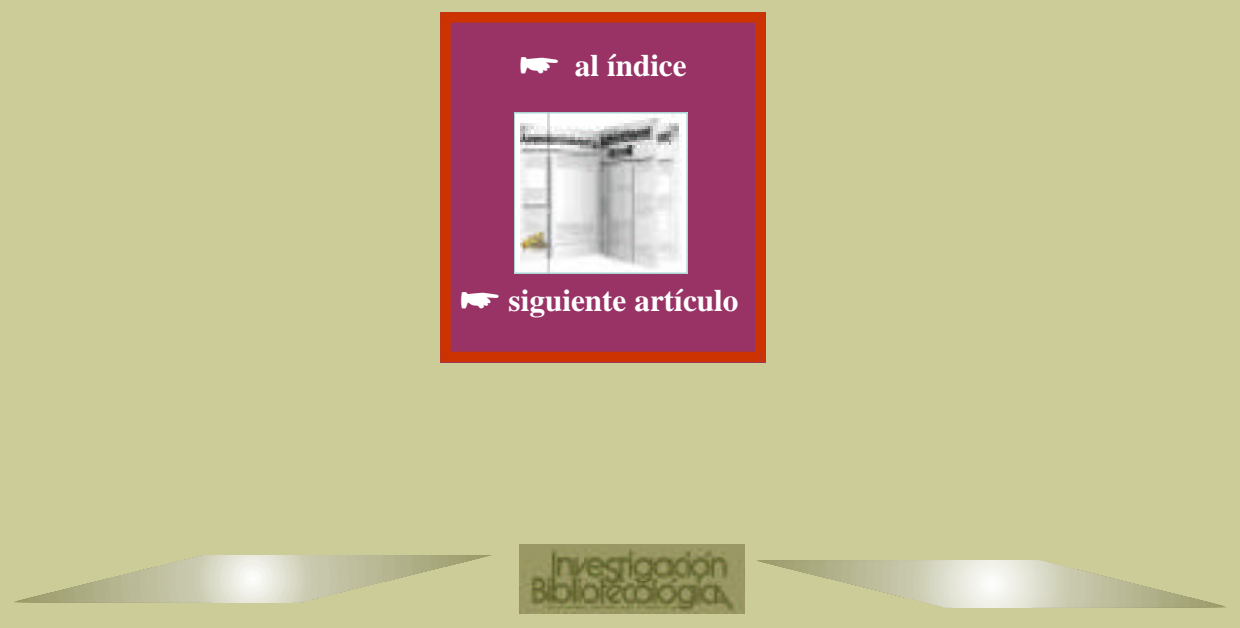